DOI: $10.1002 /($ adma.201405147)

Article type: Communication

\title{
Strongly Bias-Dependent Tunnel Magnetoresistance in Manganite Spin Filter Tunnel Junctions
}

Bhagwati Prasad, * Wenrui Zhang, Jie Jian, Haiyan Wang and Mark G. Blamire

B. Prasad, Prof. M.G. Blamire

Department of Materials Science and Metallurgy, University of Cambridge, 27 Charles Babbage Road, Cambridge, CB3 0FS, UK

E-mail: bp337@cam.ac.uk (Corresponding author)

W. Zhang, J. Jian, Dr. H. Wang

Department of Electrical and Computer Engineering

Texas A\&M University, College Station, Texas, 77843-3128, USA

Keywords: Spin filter; Manganites; Tunnel magnetoresistance; Fowler-Nordheim tunneling; Coulomb blockade

The quantum phenomenon of tunneling through an ultrathin insulating material sandwiched between conducting electrodes enables novel electronic devices with profound technological implications. ${ }^{[1-4]}$ Integrating magnetic materials into these devices has added a new dimension to electron tunneling, exploiting the spin degree of freedom, an aspect of the field of spintronics. ${ }^{[5,6]}$ The magnetic material could either be the conducting electrodes as in magnetic tunnel junctions $(\mathrm{MTJs})^{[7,8]}$ or a ferromagnetic insulator (FMI) barrier in spin filter tunnel junctions (SFTJs). ${ }^{[9-11]}$ Unlike the insulator barrier in a conventional MTJ, the FMI barrier in a SFTJ provides spin dependent barrier heights for the tunneling of the electron due to an exchange splitting in the FMI layer. $^{[9]}$ Since the tunneling probability is exponentially dependent on the barrier height, a SFTJ generates highly spin-polarized current. Although the spin polarization of the tunneling current in a SFTJ can be inferred from the temperature dependence of the junction resistance, ${ }^{[10,12]}$ direct 
detection requires a magnetic counter-electrode that has a spin-dependent density of states (DOS) at the Fermi level. Hence, the effectiveness of a spin-filter (SF) material can be determined by observing the tunnel magnetoresistance (TMR) in a quasimagnetic tunnel junction (QMTJ), consisting of a NM/SF/I/FMM structure where NM (FMM) is a normal metal (ferromagnetic metal) electrode and $\mathrm{I}$ is a nonmagnetic insulating layer, included to eliminate the magnetic exchange coupling between SF and FMM layers. Well-defined TMR responses have been realized in QMTJs utilizing the europium chalcogenides, ${ }^{[13,14]}$ ferrites, ${ }^{[11,15,16} \mathrm{La}_{1-\mathrm{x}} \mathrm{Ba}_{\mathrm{x}} \mathrm{MnO}_{3}(\mathrm{x}=1 \text { and } 0.9)^{[17,18]}$ and $\operatorname{Pr}_{0.8} \mathrm{Ca}_{0.2} \mathrm{Mn}_{1-\mathrm{y}} \mathrm{Co}_{\mathrm{y}} \mathrm{O}_{3},{ }^{[19]}$ as $\mathrm{SF}$ materials. Investigation of the bias dependence of the TMR response of the QMTJ is essential both to determine the suitable operating bias voltage regime for better device performance and to understand the details of the transport processes. However, the bias-dependence of the TMR response in these studies is rarely reported: in the QMTJs of $\mathrm{Al} / \mathrm{EuS} / \mathrm{Al}_{2} \mathrm{O}_{3} / \mathrm{Co} / \mathrm{CoO}^{[14]}$ and $\mathrm{Pt} / \mathrm{CoFe}_{2} \mathrm{O}_{4} / \mathrm{Al}_{2} \mathrm{O}_{3} / \mathrm{Co}^{[16]}$ show the TMR first gradually increasing up to a certain value with increasing bias voltage and then decreasing at higher voltages, which is in agreement with the theoretical model proposed by Saffarzadeh. ${ }^{[20]}$ On the contrary, the TMR value for $\mathrm{NiFe}_{2} \mathrm{O}_{4}{ }^{[21]} \mathrm{SF}$ material decrease monotonically as the bias voltage increases. The biasdependent TMR response of manganite FMIs ${ }^{[12,19]}$ is yet to be explored.

In this letter, we report a novel bias-dependent TMR response of the FMI manganite, $\mathrm{Sm}_{0.75} \mathrm{Sr}_{0.25} \mathrm{MnO}_{3}(\mathrm{SSMO})$-based QMTJs with conducting ferromagnetic manganite electrodes, $\mathrm{La}_{0.7} \mathrm{Sr}_{0.3} \mathrm{MnO}_{3}$ (LSMO) or $\mathrm{La}_{0.7} \mathrm{Ca}_{0.3} \mathrm{MnO}_{3}$ (LCMO), as the spin detector. The ferromagnetic insulating nature of the ultrathin $(5 \mathrm{~nm})$ SSMO thin film has already been verified earlier. ${ }^{[12]}$ In order to magnetically decouple the ferromagnetic electrode (LSMO or LCMO) and the ferromagnetic insulating (SSMO) layer, an ultrathin $(\sim 1 \mathrm{~nm})$ layer of $\mathrm{SrTiO}_{3}(\mathrm{STO})$ is inserted between them. For the relatively thin $(4-5 \mathrm{~nm})$ SSMO barriers, the dependence of the TMR is similar to the theoretical model proposed by Saffarzadeh ${ }^{[20]}$ however, for larger $(7-8 \mathrm{~nm})$ barrier thicknesses, the TMR bias dependence is more complex and indeed reverses sign at higher bias 
voltage. This is interpreted in terms of a two-stage tunneling process through an inhomogeneous FMI barrier.

Multilayer oxide thin films were grown by pulsed laser deposition (PLD) in $\mathrm{N}_{2} \mathrm{O}$ atmosphere (for details, see experimental section). The epitaxial and highly crystalline growth of these films on single crystal STO (001) substrates was confirmed by both X-ray diffraction (XRD) (see Figure S1 in supplementary information) and high-resolution transmission electron microscopy (HRTEM) investigations. Figure $1 \mathrm{a}$ shows a cross-sectional TEM image of a $\mathrm{LaNiO}_{3}(\mathrm{LNO}) / \mathrm{SSMO}(30) / \mathrm{STO}(2) / \mathrm{LSMO}$ multilayer film (the numbers in the brackets used here and later in the paper indicate the film thicknesses in nanometers). The thicknesses of the SSMO and STO barrier layers were estimated from the deposition rate of the films determined by X-ray reflectivity measurement of relatively thick films, and further confirmed by cross-sectional HRTEM. The interfaces between LSMO/STO and STO/SSMO appear sharper than the interface between SSMO/LNO; this can be seen more clearly in the cross sectional scanning transmission electron microscopy (STEM) Z-contrast image (Figure 1b), which indicates abrupt transitions at the LSMO/STO and STO/SSMO interfaces, but the SSMO/LNO interface does not seem so well defined. The multilayer oxide films were then structured into nanopillar tunnel junctions of area around $500 \times 500 \mathrm{~nm}^{2}$ by using photolithography and focused ion beam (FIB) milling. Details of the device fabrication procedure can be found elsewhere ${ }^{[22,23]}$ and in the experimental section. A cross-sectional scanning electron microscopy (SEM) image and a schematic diagram of such a nanopillar tunnel device are shown in Figure 1c and 1d, respectively. The thickness of the STO layer was kept constant $(1 \mathrm{~nm})$ and the SSMO layer thickness was changed in order to study the thickness-dependence of the transport and TMR response of the devices. 


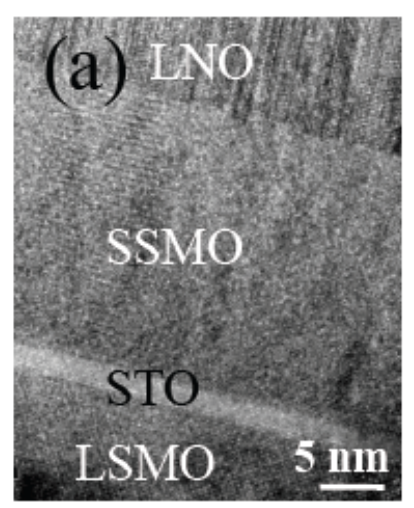

(c)

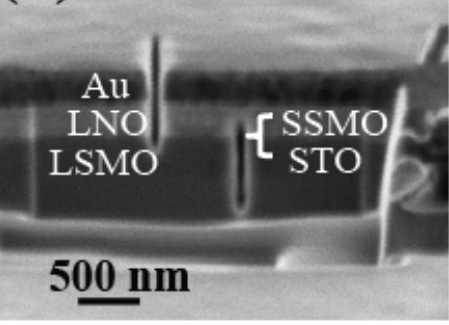

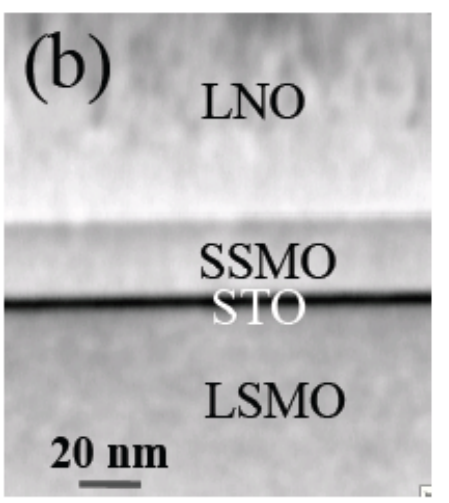

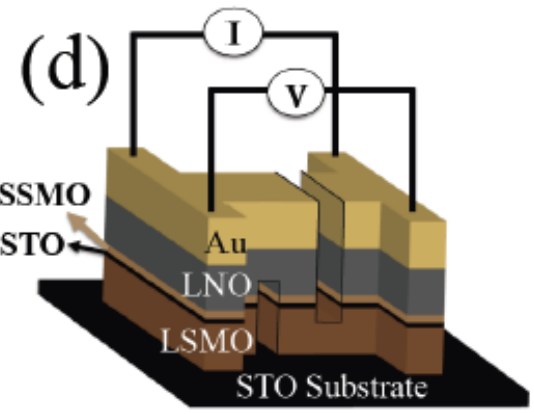

Figure 1. a) Cross-sectional TEM and b) STEM image of a LNO/SSMO $(\sim 30) / \operatorname{STO}(\sim 2) / \mathrm{LSMO}$ multilayer oxide thin film grown on STO (001) substrate. c) Cross sectional SEM image and d) schematic representation of the quasimagnetic nanopillar tunnel junction.

The transport properties of fabricated devices were measured using a four-point current-bias technique in a pulse-tube closed-cycle cryostat system. Figure $\mathbf{2 a}$ a shows the dynamic conductance curves of a LNO/SSMO(4)/STO/LSMO QMTJ, obtained by numerically differentiating the experimental $I-V$ curves measured at different temperatures. At relatively high temperatures $(100 \mathrm{~K}$ and $150 \mathrm{~K}$ ), the dynamic conductance curves are quasi-parabolic, which is one of the hallmark characteristics of a tunnel junction. ${ }^{[24]}$ The mean barrier height of the tunnel junction is $0.8 \mathrm{eV}$ determined by fitting the $I-V$ curve to the Simmons model ${ }^{[25]}$ at higher temperatures (above the $T_{C}$ $(100 \mathrm{~K})$ of SSMO barrier). This is a typical barrier-height for FMI manganite barriers. ${ }^{[12,26]}$ At low temperature (5 K), the $d I / d V$ curve of this device exhibits a reduced conductance zero bias anomaly 
(ZBA), which can be explained by the Kondo scattering due to the presence of the spin-disorder clusters at the interfaces, similar to our earlier study in $\mathrm{LNO} / \mathrm{SSMO}(5) / \mathrm{LNO}$ tunnel junctions. ${ }^{[12]}$
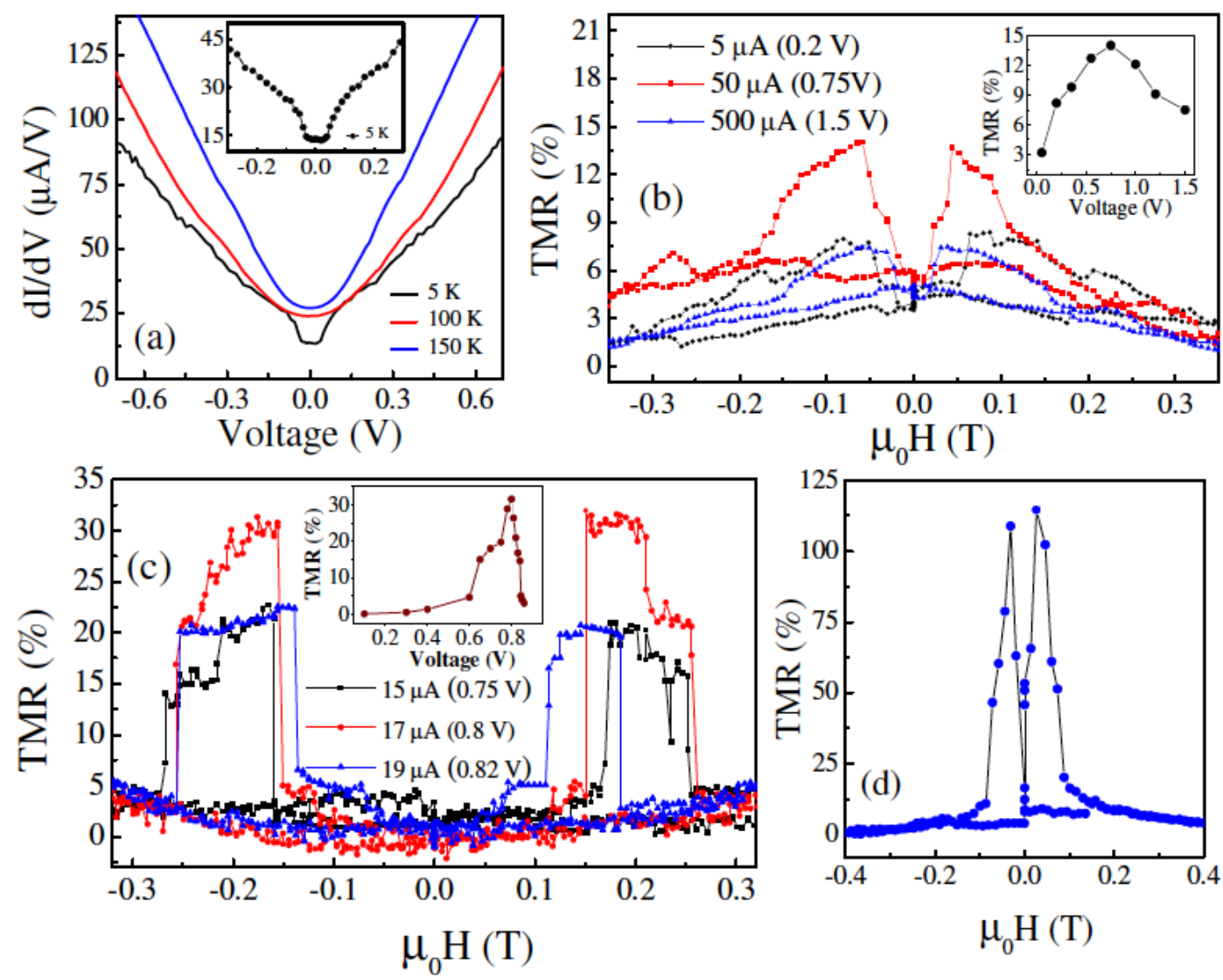

Figure 2. a) Dynamic conductance (dI/dV vs. V) curves of a Au/LNO/SSMO $(\sim 4) / \mathrm{STO} / \mathrm{LSMO}$ QMTJ device at various temperatures. The inset shows the enlarged view of the zero bias anomaly at $5 \mathrm{~K}$. b) TMR responses of the device measured at $5 \mathrm{~K}$ with varying bias. c) TMR responses of a QMTJ with LCMO electrode and $\sim 5 \mathrm{~nm}$ of SSMO barrier layer measured at $5 \mathrm{~K}$ with varying bias, indicate the degree of reproducibility. The insets of Figure b) and c) show the bias dependent TMR responses of these devices. d) TMR response of a QMTJ with LSMO electrode and $\sim 5 \mathrm{~nm}$ SSMO barrier layer at $5 \mathrm{~K}$, represents the highest observed TMR value in our devices.

Spin-dependent tunneling of the electrons through the SSMO tunnel barrier can be verified by observing the TMR response of the QMTJs. The TMR ratio of the device is defined as $\left(R_{A P}-R_{P}\right) / R_{P}$ where $R_{A P}$ and $R_{P}$ are the tunnel resistance when the magnetization of the SSMO ferromagnetic insulator layer and the LSMO ferromagnetic electrode are aligned antiparallel and parallel, 
respectively. The magnetic field was applied along the STO [100] (in-plane) direction. Figure 2b shows the TMR versus magnetic field curves of a LNO/SSMO(4)/STO/LSMO QMTJ at $5 \mathrm{~K}$ measured with various applied biases. The independent magnetization reversals of SSMO and LMSO layers are achieved by inserting $1 \mathrm{~nm}$ of STO layer between them (see Figure S2 in supporting information). With increasing bias, the TMR first increases gradually to the peak value and then decreases (see inset of Figure $2 b$ ). This is in contrast with conventional MTJs, ${ }^{[8]}$ where the TMR value decreases continuously from the maximum value at zero bias with increasing bias voltage. The peak TMR value in our device is observed at $\sim 0.75 \mathrm{~V}(50 \mu \mathrm{A})$, which is close to barrier height. In order to check the degree of reproducibility, we fabricated and measured several SSMO-based devices within same thickness range $(4-5 \mathrm{~nm})$ of the barrier layer and found similar behavior. We also measured devices with LCMO as the spin detector electrode. Figure 2c shows the representative TMR responses of one such device with $\sim 5 \mathrm{~nm}$ thick SSMO barrier layer and LCMO electrode. The smaller magnetic field at which the resistance increases sharply to high-level value corresponds to the magnetic reversal of LCMO electrode, whereas the higher magnetic field at which the resistance drops back to low-level value is close to value at which the magnetization loop of the SSMO film closes. The bias-dependent TMR response of this device (see inset of Figure 2c) shows a qualitatively similar trend as for the device with $4 \mathrm{~nm}$ thick barrier and LSMO electrode. These observed trends of the bias-dependent TMR responses in our devices with relatively thin FMI barrier $(4-5 \mathrm{~nm})$ layer are close to what Saffarzadeh $^{[20]}$ has theoretically proposed for a SFTJ as discussed later. The highest observed TMR value in our one such device with thin $(\sim 5 \mathrm{~nm})$ barrier (SSMO) and LSMO electrode is $\sim 115 \%$ (see Figure $2 \mathrm{~d}$ ). The evidence of the spin-filtering effects in these junctions was also confirmed by the temperature dependence of the junction resistance measurements (see Figure S3 in supporting information).

The transport phenomena for the thicker barriers $(7-8 \mathrm{~nm})$ are more unusual. The dynamic conductance curves of a $\mathrm{LNO} / \mathrm{SSMO}(\sim 8) / \mathrm{STO}(\sim 1) / \mathrm{LSMO}$ QMTJ at relatively higher temperatures $(>100 \mathrm{~K})$ show the conventional quasi-parabolic shape (see Figure 3a) as for the QMTJs with 

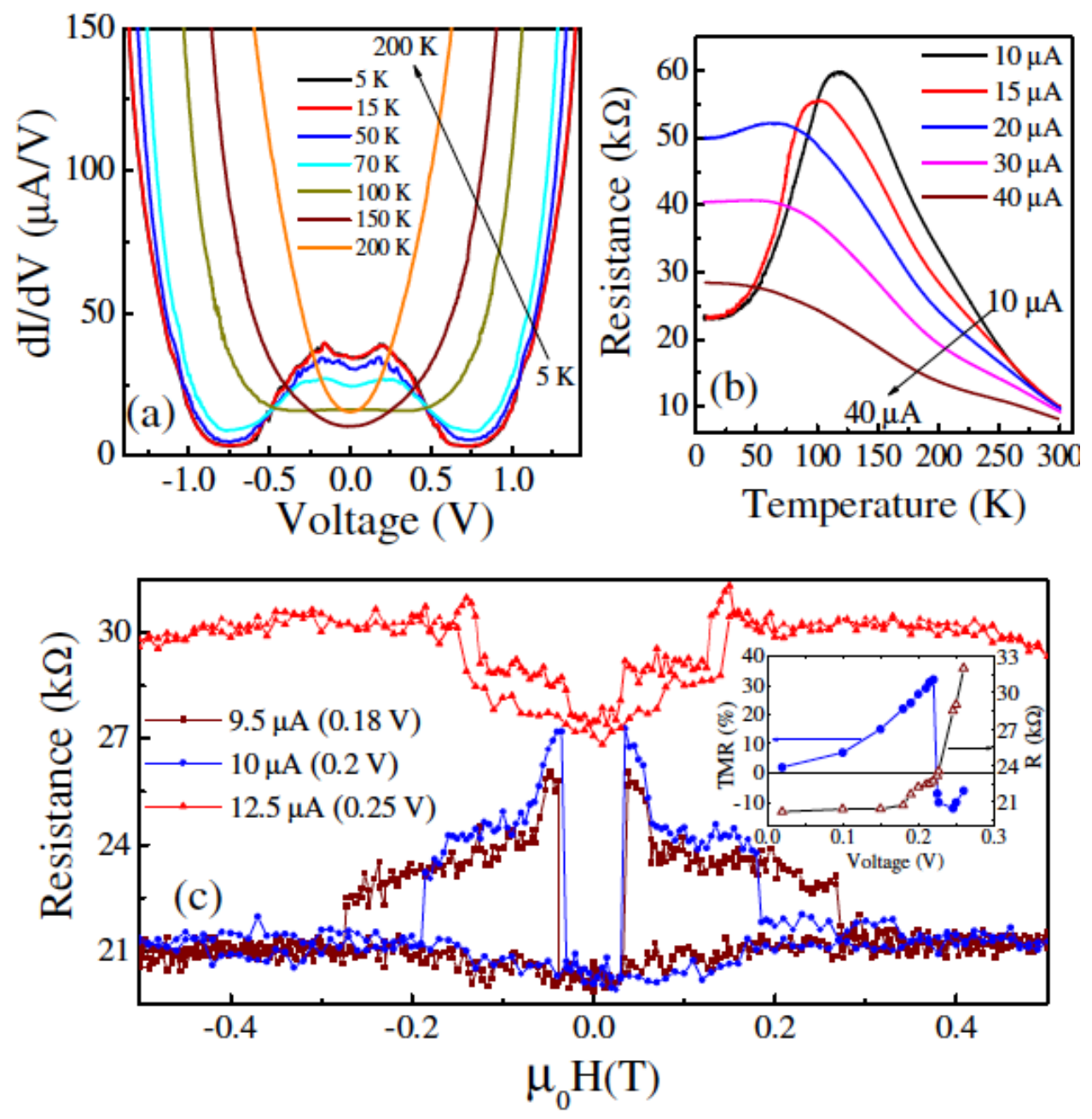

Figure 3. a) Dynamic conductance versus voltage, and b) resistance versus temperature plots of an $\mathrm{Au} / \mathrm{LNO} / \mathrm{SSMO}(\sim 8) / \mathrm{STO} / \mathrm{LSMO} \mathrm{QMTJ}$ device measured at various temperatures and biases, respectively. c) Magnetoresistance cycle for the device at $5 \mathrm{~K}$ with varying bias. The inset shows the bias dependent TMR response of the device and corresponding changes in junction resistance at the magnetic field of $0.5 \mathrm{~T}$.

thinner barrier. However, at low temperatures, $d I / d V$ versus $V$ plots of this device exhibit lower-bias peaks on either sides of zero bias instead of the large ZBA seen for thinner barriers. In order to rule out the role of the electrodes in these observed features, we also measured the $d I / d V$ versus $V$ characteristics of LNO/SSMO(8)/LNO tunnel devices (see Figure S4 in supplementary information) and found similar behavior. The dynamic conductance curve at low temperature for thicker barrier can be considered as a zero bias conductance peak for a SFTJ with a small dip at zero bias. Pal et 
$a l .{ }^{[27]}$ have reported a zero bias conductance peak at low temperature in GdN-based SFTJs. The low bias conductance peak at relatively low temperatures for the thicker barrier in our devices gradually smears with increasing temperature, which can be correlated with the disappearance of ferromagnetism and spin-filtering in SSMO barrier at $T_{C}$. The decreasing trend of the low bias conductance with increasing bias voltage at low temperatures suggests an electric-field dependent modification $^{[27]}$ of the magnetic state of SSMO layer and is confirmed by the resistance versus temperature plots of the devices at various current biases (Figure $3 b$ ). This is discussed further below.

Figure $3 \mathrm{c}$ shows the resistance versus magnetic field of a $\mathrm{LNO} / \mathrm{SSMO}(8) / \mathrm{STO} / \mathrm{LSMO}$ device at $5 \mathrm{~K}$, measured with varying bias. Similar to the thinner barrier devices, the TMR value does not evolve significantly at low bias; however, with increasing bias, the TMR gradually increases to a certain value and then suddenly drops to a negative value at higher bias, which can be correlated with the sharp increase of junction resistance at higher bias (see inset of Figure 3c). This suggests the presence of impurity states in the barrier (SSMO) layer as discussed below. ${ }^{[28,29]}$ We have also observed such unusual tunneling transport properties in other devices (see Figure S5 in supporting information).

The results presented in this paper show a rich variety of novel effects. In the remainder of the paper we discuss the possible origin of the various effects seen in our devices. The spin-filtering efficiency we have estimated in our earlier study ${ }^{[12]}$ by $\sim 5 \mathrm{~nm}$ thick SSMO barrier from the resistance versus temperature plot of a $\mathrm{LNO} / \mathrm{SSMO} / \mathrm{LNO}$ device is $\sim 75 \%$ at $5 \mathrm{~K}$. Here, we can also calculate the spin-filtering efficiency of the SSMO barrier from the TMR measurements in QMTJs by using the extended Jullière model, in which the TMR $=2 P_{S S M O} P_{F M M} /\left(1-P_{S S M O} P_{F M M}\right)$, where $P_{S S M O}$ and $P_{F M M}$ are the efficiencies of spin filtering of SSMO layer and spin polarization of the ferromagnetic electrode, respectively. The approximate spin polarization of LSMO and LCMO electrode at the interface with STO in our devices can be estimated from the TMR response of a LSMO/STO/LCMO MTJ (see Figure S6 in supplementary information) fabricated in the same way 
as the QMTJs. The optimum TMR in these MTJ devices is $\sim 225 \%$ and, assuming equal spin polarization of LSMO and LCMO, this gives $P_{L C M O}=P_{L S M O} \sim 73 \%$. Using this, the Jullière model then yields $P_{S S M O} \sim 49 \%$ for the highest observed TMR $(\sim 115 \%)$ value in a QMTJ (Figure $\left.2 \mathrm{~d}\right)$. This is smaller than the values obtained from $\mathrm{LNO} / \mathrm{SSMO} / \mathrm{LNO}$ devices, ${ }^{[12]}$ which is likely to be due to the absence of the sharp magnetic switching in SSMO layer, so that the device never attains the perfect antiparallel magnetization state.

The bias-dependence of the TMR responses of thinner $(4-5 \mathrm{~nm})$ barrier-based devices (see inset of Figure $2 \mathrm{~b}$ and $2 \mathrm{c}$ ) can be explained within the framework of spin filtering. At low bias, the spin-filtering efficiency of a SFTJ is determined by the spin-dependent barrier height (Figure 4a) and the complete suppression of the TMR at zero bias could be due to the Kondo scattering. ${ }^{[12,30]}$ The TMR value increases with increasing applied bias and exhibits the maximum at a bias corresponding to the voltage close to the barrier height. At this bias voltage, the tunneling probability of the spin-up electrons is expected to increase dramatically due to the onset of FowlerNordheim (F-N) tunneling, while the spin-down electrons tunnel probability is still determined by direct tunneling, and thereby the device exhibits a large increase in TMR (Figure 4b). With further increase in the bias voltage, the Fermi level of the source electrode may cut both spin-up and spindown bands. As a result, the tunneling probability increases for both spin(s) electrons, and hence the TMR value decreases (Figure 4c). The transition from direct tunneling to F-N tunneling in our devices can be confirmed from the $I-V$ characteristics of a $\mathrm{LNO} / \mathrm{SSMO}(\sim 5) / \mathrm{STO} / \mathrm{LCMO}$ device for the parallel and antiparallel configurations measured at $5 \mathrm{~K}$ (Figure $4 \mathrm{~d}$ ). The sharp increase in current at higher bias clearly indicates the onset of the F-N tunneling, where the crossover from direct tunneling to F-N tunneling appears earlier in parallel than antiparallel configuration. The dependence of the $I-V$ curve on the relative orientation of the magnetizations of SSMO and LCMO layers also corroborates the evidence of spin-filtering effect. The extracted exchange splitting value is about $\sim 60 \mathrm{meV}$. 

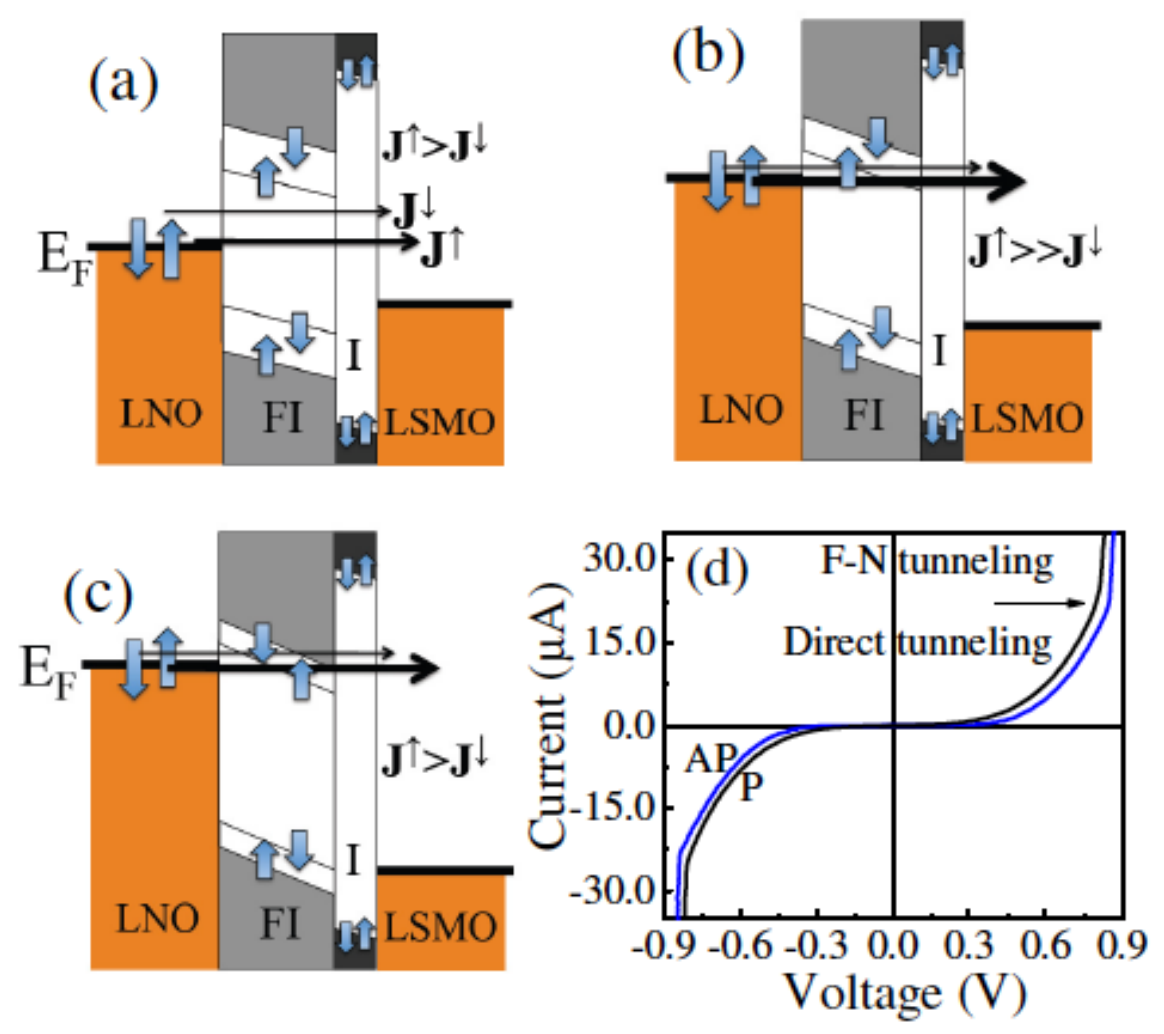

Figure 4. Schematic view of the band diagram of a QMTJ at a) low, b) intermediate, and c) higher bias regimes indicate that the mode of transport of electrons across the junction is direct tunnelling at low bias regime, and FN tunnelling for spin-up electrons at intermediate bias and both spin-up and spin-down electrons at higher bias regimes. d) I-V characteristic of an $\mathrm{Au} / \mathrm{LNO} / \mathrm{SSMO}(\sim 5) / \mathrm{STO} / \mathrm{LCMO}$ device at $5 \mathrm{~K}$ for parallel and antiparallel configurations. The arrow indicates the onset of the F-N tunnelling at higher bias.

Although SSMO is found to be in a homogeneous ferromagnetic state in a narrow range of the strontium concentration, ${ }^{[31]}$ the existence of the nanoclusters of secondary phases (ferromagnetic metallic (FMM) and/or antiferromagnetic insulating) may not be ruled out. ${ }^{[12,31]}$ The inhomogeneity in thinner barrier is expected to be negligible, hence most of the electrons tunnel directly through the barrier and as a result, the tunnel device exhibits the conventional SFTJ behavior. However, the possibility of the phase-separated metallic nanoclusters present in barrier layer will increase with increasing barrier thickness. The charging effect of these clusters could give rise to Coulomb blockade (CB) phenomena in our devices. The small dip observed at zero bias in the dynamic 
conductance curves for the fabricated devices with thicker barrier (SSMO) at low temperature regime can be associated with the Coulomb gap caused by the FMM nanoclusters present in the barrier layer. ${ }^{[32,33]} \mathrm{CB}$ effects have been widely observed in phase-separated manganites systems. ${ }^{[34-36]}$ The charging energy $\left(E_{C}\right)$ in our device is assumed to be nearly equal to the half of the width of dip, which is estimated to be in the order of $100 \mathrm{meV}$ at $5 \mathrm{~K}$. With considering the spherical shape of these FMM nanoclusters, their average diameter $(d)$ can be calculated by using the relationship of $E_{C}=\frac{e^{2}}{2 C}\left(\frac{e^{2}}{4 \pi \varepsilon_{0} \varepsilon d}\right),{ }^{[28]}$ where $C$ is the capacitance of a FMM nanocluster, $\varepsilon$ and $\varepsilon_{0}$ are the relative permittivity of the barrier and the permittivity of the vacuum, respectively. Using the above value of $E_{C}$ and $\varepsilon(10)$ for manganites, ${ }^{[37]}$ the value of $d$ is then obtained to be $\sim 1.4 \mathrm{~nm}$. The absence of the CB staircase phenomenon in our devices is due to the relatively large value of $E c$ of the FMM nanocluster, i.e., a large voltage is required to bring the electronic states of the nanoclusters in resonance with the Fermi levels of the electrodes. When the applied bias voltage is large enough to overcome the charging energy associated with $\mathrm{CB}$ effect, the transport across the barrier is dominated by the sequential tunneling through the FMM nanoclusters. The bias-dependent TMR response at lower bias in our thicker barrier-based device shows a similar trend as the thinner barrier-based devices, which can also be explained within the framework of spin filtering. Since the sequential tunneling in small bias regime is suppressed, the tunneling of the electron through the FMI barrier is expected to show the conventional spin filtering effect. The negative value of the TMR at higher bias might be due to the sequential tunneling of the electron through the spin accumulation states in the barrier region. The possibility of the spin accumulation in half metallic manganite ferromagnetic nanoclusters is high due to the large difference in the DOS for majority and minority spins at Fermi level. Moreover, the role of the oxygen vacancies and associated defects presence in FMI barrier can not be ruled out for these unusual tunneling transport behaviors.

In addition to the small dip at zero bias in $d I / d V$ curves at low temperatures, the lower bias conductance peak is also correlated with the thickness of the barrier layer. The decrease in conductance with increasing bias voltage implies the suppression of the exchange splitting in barrier 
regime with increasing bias voltage. The decreasing nature of the spin polarization with increasing bias has also been observed in $\mathrm{LNO} / \mathrm{SSMO}(\sim 5 \mathrm{~nm}) / \mathrm{LNO}$ SFTJs at higher bias. ${ }^{[12]}$ Such an electricfield-dependent spin polarization in FMI barrier layer was first reported in GdN-based SFTJs. ${ }^{\text {[27] }}$ As seen in Figure 3b, with increasing bias, the peak of $R$ vs $T$ curve shifts towards lower temperatures, as well as the extent of fall of resistance at low temperatures decreases. This indicates that the suppression of the spin-filtering efficiency at higher bias is correlated with the corresponding decrease in conductance. The insignificant drop in resistance at applied current bias of $30 \mu \mathrm{A}$ at low temperatures (below $30 \mathrm{~K}$ ) corresponds to the dip in dynamic conductance curve at intermediate voltage regime $(\sim 0.6 \mathrm{~V})$. Moreover, there is no low-temperature drop in resistance observed at higher current $(40 \mu \mathrm{A})$ bias.

The peak in the $R-T$ curve at lower bias $(10 \mu \mathrm{A})$ for thicker barrier-based device is observed at a temperature higher than the bulk $T_{C}$ of the SSMO (see Figure $3 \mathrm{~b}$ ). Such increase of the $T_{C}$ of the ultrathin SSMO layer could be due to the presence of the oxygen vacancies. ${ }^{[38]}$ These oxygen vacancies can be moved towards the interface by large applied electric field across the barrier layer. As a consequence, the net ferromagnetism and $T_{C}$ of the SSMO layer are expected to decrease with increasing applied bias. ${ }^{[39]}$ Moreover, the spin scattering events or direct tunneling without spin filtering caused by the defect states present in the barrier and/or at the interfaces could decrease the spin polarization of the tunnel device at higher bias. ${ }^{[1]}$

Yang et al. ${ }^{[40]}$ have observed the double-peak structure as ZBA in the dynamic conductance curves of planar MTJs with magnetic nanodots embedded in MgO tunnel barriers. Such ZBA is explained by the spin-split due to the magnetic exchange interaction between the magnetic nanodots and the ferromagnetic electrodes. However, the novel zero bias conductance behavior in our devices is observed even in the absence of ferromagnetic electrodes (LNO/SSMO/LNO tunnel junction), and thereby discounts the role of Kondo resonance tunneling in our devices. It is interesting to note that the inhomogeneous phase found to be accountable for the anomalous behavior of the relatively thick barrier-based tunnel devices was undetectable by the standard structural, magnetic and 
transport investigation of this material. Therefore this indicates the high level of sensitivity of the spin-dependent tunneling to the minute defects or inhomogeneity present in the barrier layer.

In summary, we have shown that the TMR responses of $\mathrm{Sm}_{0.75} \mathrm{Sr}_{0.25} \mathrm{MnO}_{3}$-based SFTJs are strongly bias-dependent with two different behaviors in two different thickness regimes of the FMI barrier. For relatively thin $(4-5 \mathrm{~nm})$ barriers, the TMR value first increases to the peak value with increasing the bias and then it decreases at higher bias. This has been successfully explained within the standard framework of spin filtering in which the maximum TMR value at the intermediate bias has been attributed to the onset of spin-dependent Fowler-Nordheim (F-N) tunneling through the spin-polarized conduction band of SSMO barrier. On the other hand, for thicker barriers (7-8 nm), the TMR goes to the negative value at higher bias. This is interpreted in terms of sequential tunneling of electrons through the inhomogeneous SSMO barrier layer; a process correlated by Coulomb blockade phenomenon at low bias and spin accumulation effect at higher bias. These wide varieties of novel effects shown in this paper are expected to broaden the scope of spin-filtering materials and open new opportunities for spintronic applications.

\section{Experimental Section:}

All oxides films (SSMO, STO, LNO, LCMO and LSMO) were epitaxially grown by pulsed laser deposition on STO (100) single-crystal substrates by ablating the stoichiometric ceramic targets. A KrF laser $(\lambda=248 \mathrm{~nm})$ was used at $5 \mathrm{~Hz}$ repetition rate with a fluence of $\sim 1 \mathrm{~J} / \mathrm{cm}^{2}$ on the target. The $\mathrm{N}_{2} \mathrm{O}$ partial pressure was set to $20 \mathrm{~Pa}$ for manganites (SSMO, LCMO and LSMO) and STO films with the substrate temperature of $700{ }^{\circ} \mathrm{C}$. While LNO films were grown at a $\mathrm{N}_{2} \mathrm{O}$ partial pressure of $30 \mathrm{~Pa}$ and a temperature of $650{ }^{\circ} \mathrm{C}$. After growth, the samples were cooled down to room temperature in $100 \mathrm{~Pa} \mathrm{~N}_{2} \mathrm{O}$. The phase and crystalline qualities of the deposited films were determined by using a PANalytical high resolution X-ray diffractometer with $\mathrm{Cu} \mathrm{K} \alpha$ radiation, a 2bounce hybrid monochromator and $0.5 \mathrm{~mm}$ slit beam tunnel. Polycrystalline gold layer (thickness $\sim 200 \mathrm{~nm}$ ) was deposited on the multilayer epitaxial oxide thin films by DC magnetron sputtering, which serves as a top contact and also minimize ion-beam damage from subsequent processing 
steps. A series of $4-\mu \mathrm{m}$-wide tracks were first patterned by conventional optical photolithography and then created by Ar-ion milling. Subsequently, focused ion beam (FIB) nanomachining was used to fabricate the final nanopillar device with an average dimension of $500 \times 500 \mathrm{~nm}^{2}$. The thickness of both top and bottom metallic electrode layers (LNO and LSMO or LCMO) was $~ 200$ nm. Up to maximum 14 devices were created in a chip, where each device is connected to four large area contact pads for the four point transport measurements. The electrical contacts were made by wire bonding, which ensures that the current passes from the top Au-LNO electrode to bottom ferromagnetic metallic electrode through the SSMO-STO barrier layers. Although, the current path in our device may cross the barrier twice, the resistance is dominated by the barrier in the nanopillar and the second barrier, which is formed by the lead structure is orders of magnitude larger in area and hence generates negligible voltage. The electrical measurements of the devices were performed in four-probe current biased configuration in a pulse-tube cryocooled measurement system. In our measurements, the bias direction is defined with respect to the bottom ferromagnetic electrode (i.e. positive voltage means this electrode is positive).

\section{Supporting Information}

Supporting information is available from the Wiley Online Library or from the author.

\section{Acknowledgements:}

This work was partially supported by the ERC Advanced Integrators Grant "SUPERSPIN". B.P. was funded by the Nehru Trust for Cambridge University and St John's College. The TEM work at Texas A\&M was supported by the U.S. National Science Foundation (NSF-DMR 0846504). 


\section{References:}

[1] R. W. Gurney, E. U. Condon, Nature 1928, 122, 439.

[2] L. Esaki, Phys. Rev. 1958, 109, 603.

[3] P. Bedrossian, D.M. Chen, K. Mortensen, J. A. Golovchenko, Nature 1989, 342, 258.

[4] D. M. Eigler, E. K. Schweizer, Nature 1990, 344, 524.

[5] I. Zutic, J. Fabian, S. D. Sarma, S. Das, Rev. Mod. Phys. 2004, 76, 323.

[6] G.-X. Miao, M. Munzenberg, J. S. Moodera, Rep. Prog. Phys. 2011, 74, 036501.

[7] S. Yuasa, T. Nagahama, A. Fukushima, Y. Suzuki, K. Ando, Nat. Mater. 2004, 3, 868.

[8] E. Y. Tsymbal, O. N. Mryasov, P. R. LeClair, J. Phys. Condens. Matter 2003, 15, R109.

[9] J. S. Moodera, T. S. Santos, T. Nagahama, J. Phys. Condens. Matter 2007, 19, 165202.

[10] K. Senapati, M.G. Blamire, Z. H. Barber, Nat. Mater. 2011, 10, 849.

[11] J.-B. Moussy, J. Phys. D. Appl. Phys. 2013, 46, 143001.

[12] B. Prasad, M. Egilmez, F. Schoofs, T. Fix, M. E. Vickers, W. Zhang, J. Jian, H. Wang, M. G. Blamire, Nano Lett. 2014, 14, 2789.

[13] P. LeClair, J. K. Ha, H. J. M. Swagten, J. T. Kohlhepp, C. H. van de Vin, W. J. M. de Jonge, Appl. Phys. Lett. 2002, 80, 625.

[14] T. Nagahama, T. S. Santos, J. S. Moodera, Phys. Rev. Lett. 2007, 99, 016602.

[15] U. Lüders, A. Barthélémy, M. Bibes, K. Bouzehouane, S. Fusil, E. Jacquet, J.-P. Contour, J.-F. Bobo, J. Fontcuberta, A. Fert Adv. Mater. 2006, 18, 1733.

[16] A. V. Ramos, M. -J. Guittet, J.-B. Moussy, R. Mattana, C. Deranlot, F. Petroff, C. Gatel, Appl. Phys. Lett. 2007, 91, 122107.

[17] M. Gajek, M. Bibes, A. Barthélémy, K. Bouzehouane, S. Fusil, M. Varela, J. Fontcuberta, A. Fert, Phys. Rev. B, 2005, 72, 020406(R).

[18] M. Gajek, M. Bibes, S. Fusil, K. Bouzehouane, J. Fontcuberta, A. Barthélémy, A. Fert, Nat. Mater. 2007, 6, 296.

[19] T. Harada, I. Ohkubo, M. Lippmaa, Y. Sakurai, Y. Matsumoto, S. Muto, H. Koinuma, M. Oshima, Phys. Rev. Lett. 2012, 109, 076602.

[20] A. Saffarzadeh, J. Magn. Magn. Mater. 2004, 269, 327.

[21] U. Lüders, M. Bibes, K. Bouzehouane, E. Jacquet, J.-P. Contour, S. Fusil, J.-F. Bobo, J. Fontcuberta, A. Barthélémy, A. Fert, Appl. Phys. Lett. 2006, 88, 082505.

[22] M. C. Wu, A. Aziz, J. D. S. Witt, M. C. Hickey, M. Ali, C. H. Marrows, B. J. Hickey, M. G. Blamire, Nanotechnology 2008, 19, 485305.

[23] C. Bell, G. Burnell, D.-J. Kang, R. H. Hadfield, M. J. Kappers, M. G. Blamire, Nanotechnology 2003, 14,630 .

[24] E. L. Wolf, Principles of electron tunneling spectroscopy, Oxford University Press, New York, 1985.

[25] J. G. Simmons, J. Appl. Phys. 1963, 34, 1793. 
[26] T. Harada, I. Ohkubo, M. Lippmaa, Y. Sakurai, Y. Matsumoto, S. Muto, H. Koinuma, M. Oshima, Adv. Funct. Mater. 2012, 22, 4471.

[27] A. Pal, K. Senapati, Z. H. Barber, M. G. Blamire, Adv. Mater. 2013, 25, 5581.

[28] K. Yakushijia, S. Mitanib, F. Ernultb, K. Takanashib, H. Fujimorib, Phys. Rep. 2007, 451, 1.

[29] E.Y. Tsymbal, A. Sokolov, I. F. Sabirianov, B. Doudin, Phys. Rev. Lett. 2003, 90, 186602.

[30] K. I. Lee, S. J. Joo, J. H. Lee, K. Rhie, T.-S. Kim, W.Y. Lee, K. H. Shin, B. C. Lee, P. LeClair, J.-S. Lee, J.-H. Park, Phys. Rev. Lett. 2007, 98, 107202.

[31] A.I. Kurbakov, J. Magn. Magn. Mater, , 2010, 322, 967.

[32] H. Sukegawa, S. Nakamura, A. Hirohata, N. Tezuka, K. Inomata, Phys. Rev. Lett. 2005, 94, 068304.

[33] T. B. Tran, I. S. Beloborodov, J. Hu, X. M. Lin, T. F. Rosenbaum, H. M. Jaeger, Phys. Rev. B 2008, $78,075437$.

[34] M. Garcia-Hernandez, F. Guinea, A. de Andres, J. L. Martinez, C. Prieto, L. Vazquez, Phys. Rev. B 2000, 61, 9549 .

[35] V. D. Okunev, R. Szymczak, M. Baran, H. Szymczak, and P. Gierłowski, Phys. Rev. B, 2006, 74, 014404.

[36] T. Sarkar, M. V. Kamalakar, A. K. Raychaudhuri, New J. Phys. 2012, 14, 033026.

[37] L. Balcells, J. Fontcuberta, B. Martinez, X. Obradors, Phys. Rev. B 1998, 58, R14697.

[38] R. Zhao, K. Jin, Z. Xu, H. Guo, L. Wang, C. Ge, H. Lu, G. Yang, Appl. Phys. Lett. 2013, 102, 122402.

[39] T. Fix, E.-M. Choi, J. W. A. Robinson, S. B. Lee, A. Chen, B. Prasad, H. Wang, M. G. Blamire, J. L. MacManus-Driscoll, Nano Lett. 2013, 13, 5886.

[40] H. Yang, S.-H. Yang, G. Ilnicki, J. Martinek, S. S. P. Parkin, Phys. Rev. B, 2011, 83, 174437. 
Copyright WILEY-VCH Verlag GmbH \& Co. KGaA, 69469 Weinheim, Germany, 2013.

\section{Supporting Information}

for Adv. Mater., DOI: 10.1002/( adma.201405147)

\section{Strongly Bias-Dependent Tunnel Magnetoresistance in Manganite Spin Filter Tunnel Junctions}

Bhagwati Prasad, *Wenrui Zhang, Jie Jian, Haiyan Wang and Mark G. Blamire

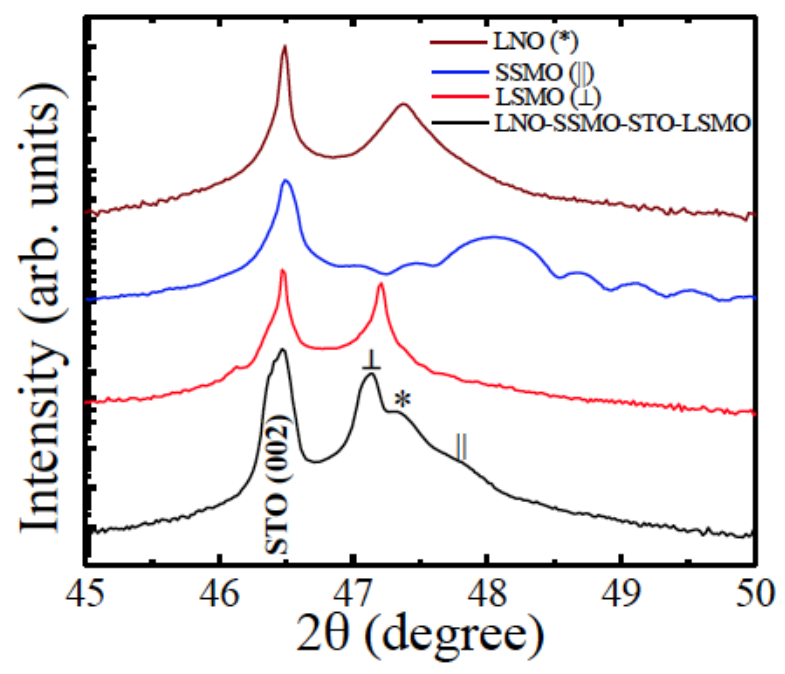

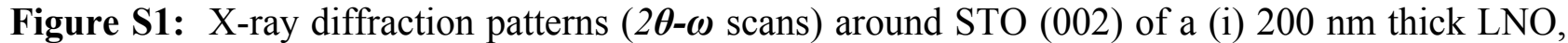
(ii) $30 \mathrm{~nm}$ thick SSMO, (iii) $200 \mathrm{~nm}$ thick LSMO and (iv) LNO(200)/SSMO(30)/STO(2)/LSMO(200) multilayer films on STO (001) substrate.

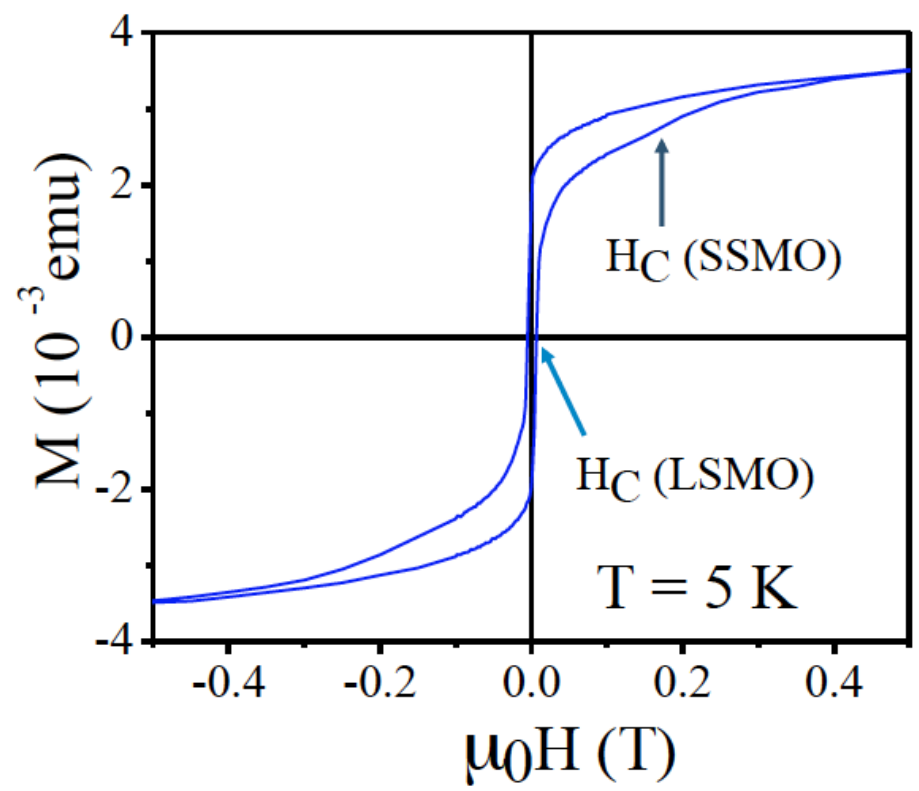

Figure S2: The M-H curve of a SSMO $(100 \mathrm{~nm}) / \mathrm{STO}(1 \mathrm{~nm}) / \mathrm{LSMO}(100 \mathrm{~nm})$ heterostructure, indicates the independent magnetization reversals of LSMO and SSMO layers. 

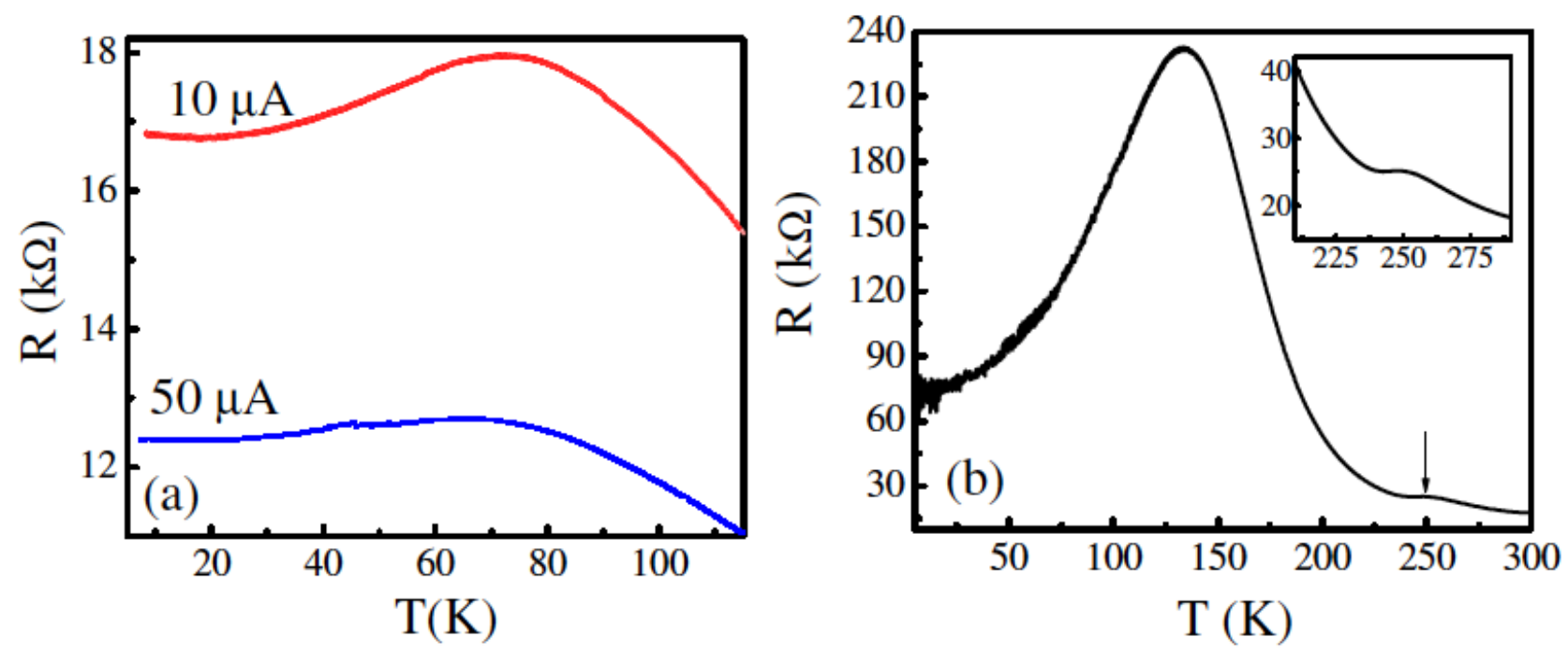

Figure S3: The temperature dependent junction resistance of a) a Au/LNO/SSMO(4)/STO/LSMO and b) a $\mathrm{Au} / \mathrm{LNO} / \mathrm{SSMO}(5) / \mathrm{STO} / \mathrm{LCMO}$ tunnel junctions. The enlarge view of the $\mathrm{R}$ vs $\mathrm{T}$ curve at higher temperatures shown in the inset of Figure $\mathrm{b}$ exhibits a little hump at $\sim 250 \mathrm{~K}$, which is corresponding to the paramagnetic to ferromagnetic transition of LCMO electrode.

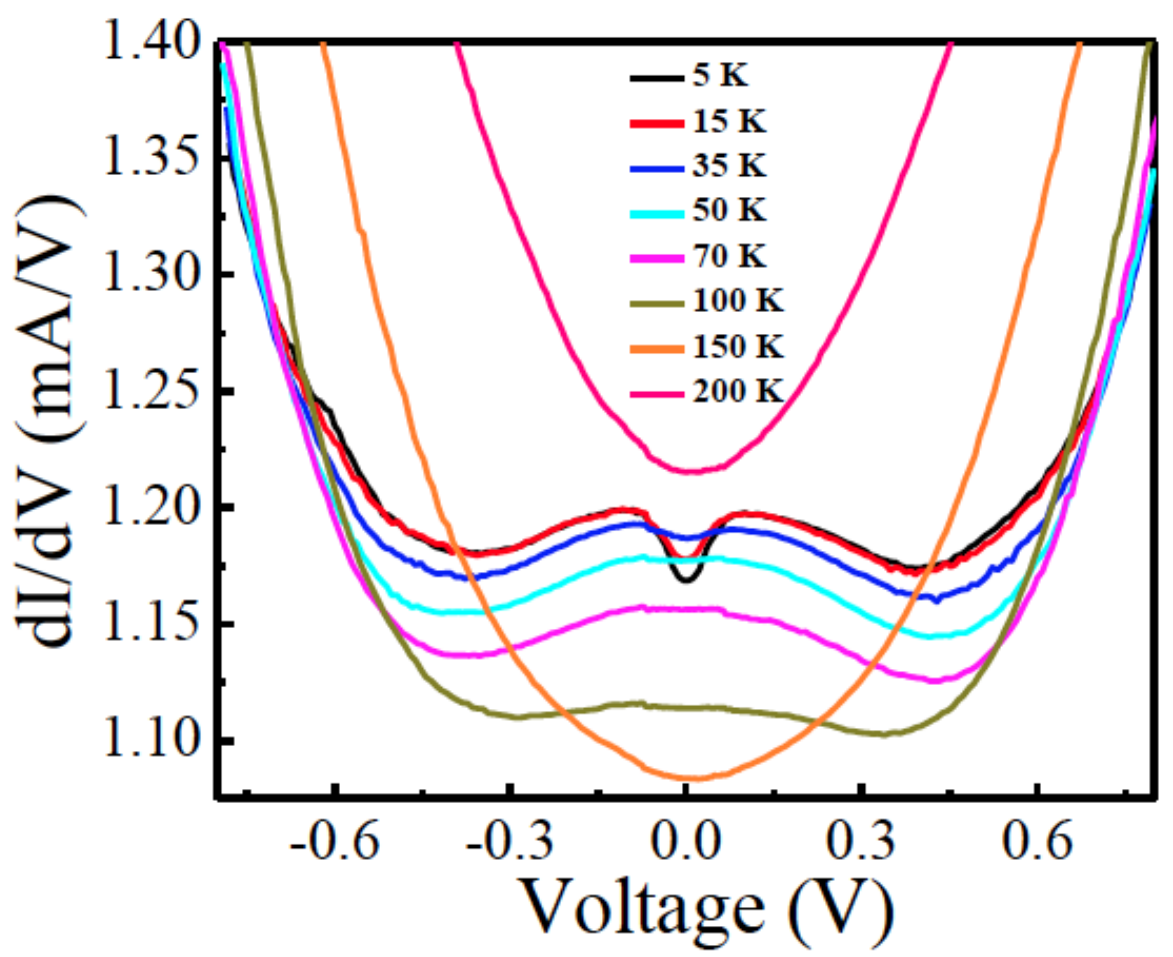

Figure S4: Dynamic conductance versus voltage plots of an Au/LNO/SSMO( 8)/LNO nanopillar tunnel device at various temperatures emphasis the presence of the novel zero bias conductance behavior even in the absence of ferromagnetic electrodes. 

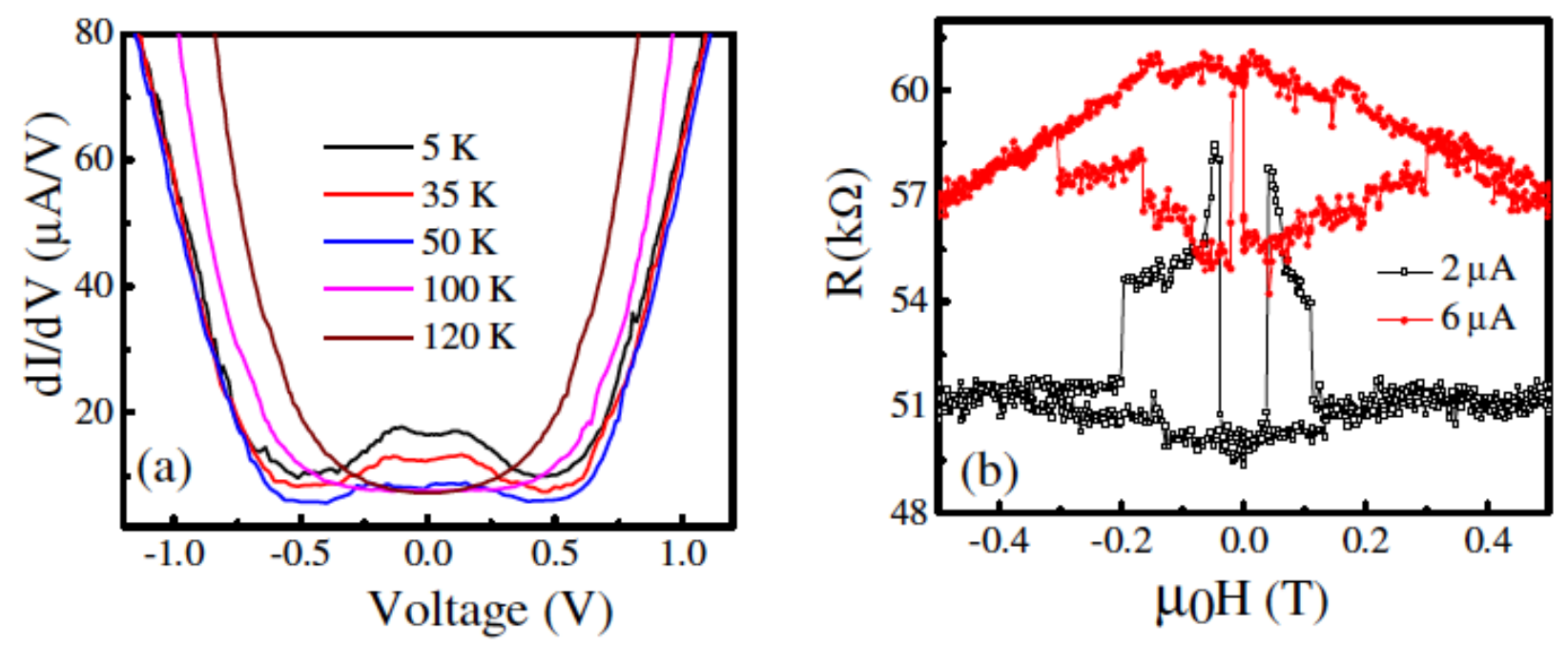

Figure S5: a) Dynamic conductance versus voltage, and b) resistance versus magnetic field plots of a different $\mathrm{Au} / \mathrm{LNO} / \mathrm{SSMO}(\sim 8) / \mathrm{STO} / \mathrm{LSMO}$ QMTJ device measured at various temperatures and biases, respectively. These results shows qualitatively similar trends as for the device mentioned earlier (Figure 3), emphasising the reproducibility of the observed effects.

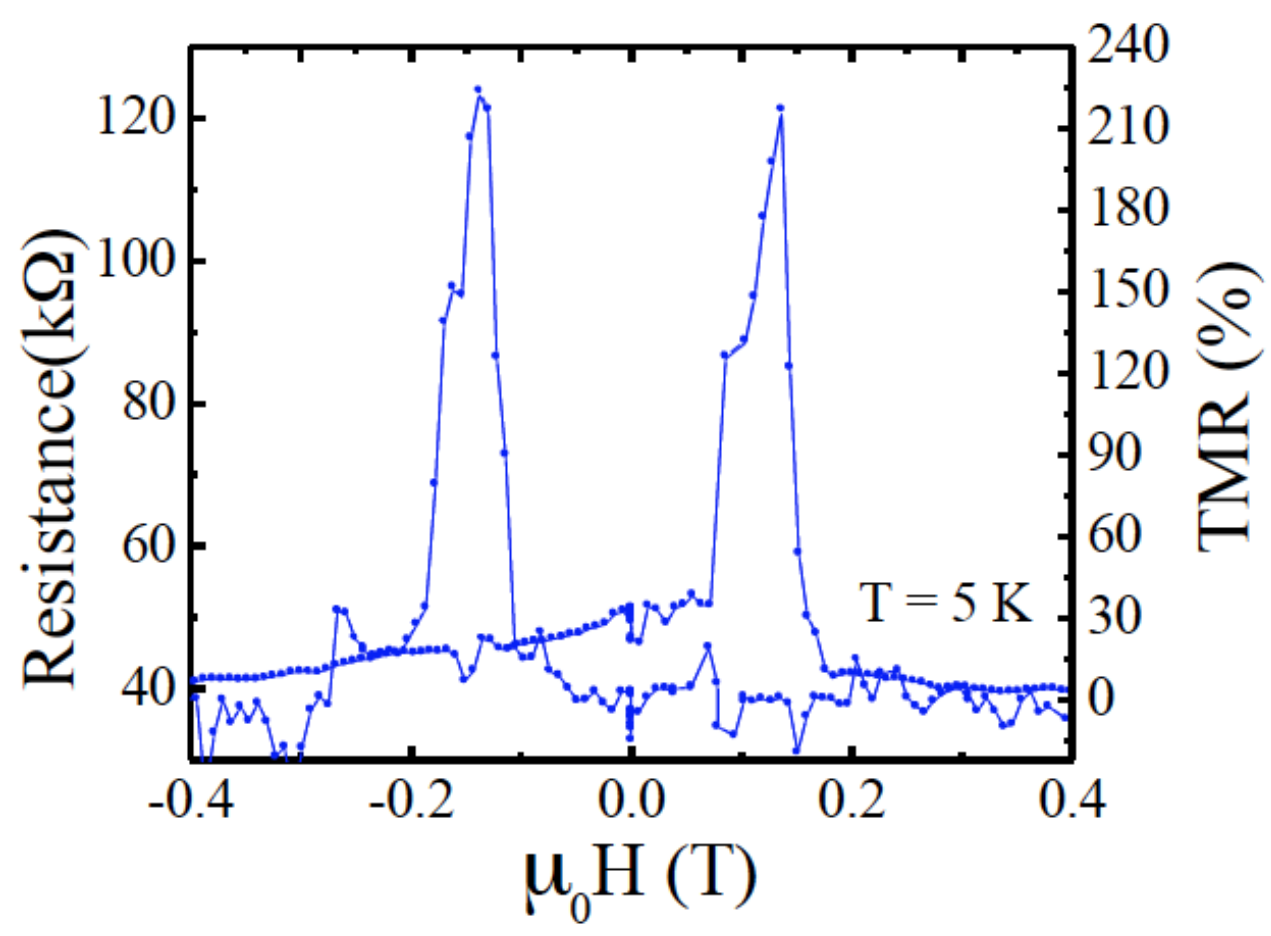

Figure S6: Magnetoresistance cycle for a Au/LCMO/STO (3)/LSMO magnetic tunnel junction at 5 $\mathrm{K}$ with $0.5 \mu \mathrm{A}$ current bias, represents the optimum TMR response $(225 \%)$ observed in our LCMO/STO/LSMO based MTJs. By using the Jullière model and considering the nearly same spin polarization of LSMO and LCMO electrodes, the corresponding spin polarization of each of these electrodes is estimated to be $\sim 73 \%$. 
